\title{
Datos experimentales de la cinética del secado y del modelo matemático para pulpa de cupuaçu (Theobroma grandiflorum) en rodajas
}

\author{
Kinetic drying experimental data and mathematical model for cupuaçu pulp (Theobroma grandiflora) slices
}

\author{
Abraham Damian GIRALDO-ZUNIGA ${ }^{1 *}$, Aroldo ARÉVALO-PINEDO², Alessandra Ferreira SILVA ${ }^{1}$, \\ Polyana Ferreira SILVA ${ }^{1}$, Juan Carlos VALDES-SERRA ${ }^{1}$, Marta Cristina de Menezes PAVLAK ${ }^{1}$
}

\begin{abstract}
Resumen
En este trabajo fueron obtenidos experimentalmente la cinética del secado de la pulpa de cupuaçu en forma de rodajas con espesor de $0,5 \mathrm{~cm}$. El secado fue realizado utilizándose un secador laboratorial de bandejas, a las temperaturas de 50,60 y $70{ }^{\circ} \mathrm{C}$ y a la velocidad del aire de secado de $1,5 \mathrm{~m} / \mathrm{s}$. De acuerdo con los datos de la cinética del secado se puede observar que cuanto mayor es la temperatura del secado mayor es la velocidad del secado. El tiempo requerido para secar el producto hasta una humedad del 20\%, fueron 9,2, 8,1 y 7,3 horas para las temperaturas de secado de 50,60 y $70{ }^{\circ} \mathrm{C}$ respectivamente. Las curvas experimentales del secado fueron ajustados al modelo difusional de Fick considerándose la muestra como una lámina infinita y al modelo de Page. Los dos modelos se ajustaron bien a los datos experimentales. Los coeficientes de difusión del modelo de Fick variaron de 1.171 a $1.561 \mathrm{~m} / \mathrm{s}^{2}$.

Palabras-clave: secagem; moelagem matemática; cupuaçu; cinética; Theobroma grandiflorum.
\end{abstract}

\begin{abstract}
This work examined experimentally the kinetics of drying cupuaçu pulp (Theobroma grandiflorum) slices. The experimental runs were driven in a laboratory dryer, at temperatures of 50,60 and $70{ }^{\circ} \mathrm{C}$ and an air drying velocity of $1.5 \mathrm{~m} / \mathrm{s}$. According to the kinetics study, increasing the temperature led to a decrease in drying time. The required drying times were 9.2, 8.1 and 7.3 hours for drying temperatures of 50, 60 and $70{ }^{\circ} \mathrm{C}$ respectively. The experimental drying curves were adjusted to Fick's diffusional model for an infinite lamina with shrinkage and Page model. The diffusion coefficients of the Fick model varied between 1.171 and $1.561 \mathrm{~m} / \mathrm{s}^{2}$.

Keywords: drying; mathematical model; cupuaçu; kinetic; Theobroma grandiflorum.
\end{abstract}

\section{Introducción}

El cupuaçu (Theobroma Grandiflorum) es una planta de mediano porte que pertenece a la familia de las esterculiáceas, la misma del Cacao y que puede alcanzar hasta $20 \mathrm{~m}$ de altura (GOMES, 2007).

La fruta del cupuaçu representa una fuente primaria de alimento para los pobladores de la floresta Amazónica del Brasil, como para los animales de la región. Esta fruta es reconocida por su pulpa cremosa y por su sabor exótico, muy utilizado en el Brasil y el Perú para la fabricación de jugos, helados, mermeladas, jaleas, entre otros productos.

La fruta tiene una forma elíptica, semejante al fruto del cacao, su pulpa es delicadamente fibrosa de sabor ácido-dulce y aroma agradable (LORENZI, 1998). Debido a las óptimas características para industrialización, además del aroma y sabor inigualables está despertando gran interés en el mercado nacional e internacional. Según Neves Filho (1994) el alto grado de descomposición en los meses de cosecha contribuye para una pérdida de aproximadamente $40 \%$ de la producción nacional de los frutos. Así como la mayoría de las frutas grande parte de la cosecha de cupuaçu es desperdiciada, ya que se comercializa sin ningún procesamiento. Por esta razón resulta muy necesario estudiar métodos de industrialización y conservación que puedan viabilizar el aprovechamiento de esta fruta, permitiendo su almacenamiento y comercialización por un período de tiempo prolongado. Las pérdidas entre la cosecha de los productos agrícolas y su transformación en alimento para el consumo varían significativamente en las diferentes etapas y están en función de la época de cosecha, variedad, enfermedades, clima, sistema de cosecha, proceso y comercialización (CHITARRA, M. I. F.; CHITARRA, A. B., 1990).

La conservación del alimento es muy importante para evitar el desperdicio, entre las técnicas de conservación de alimentos el proceso de secado representa una alternativa tecnológica para la reducción de las pérdidas pos-cosecha, pues el secado es un método simple y relativamente barato cuyo principio básico es el de reducir el contenido de agua del alimento, ocasionando una reducción drástica de la actividad de agua de los productos alimenticios, aumentando el tiempo

Recebido para publicação em 19/3/2008

Aceito para publicação em 5/1/2009 (003338)

Curso de Engenharia de Alimentos, Universidade Federal do Tocantins - UFT, Av. NS - 15, ALC NO -14, CEP 77000-000, Campus Universitario, Palmas - TO, Brasil,

E-mail: abraham@uft.edu.br

2 Engenharia de Alimentos, Universidade Federal do Tocantins - UFT, CEP 77000000, Palmas - TO, Brasil

${ }^{*}$ A quem a correspondência deve ser enviada 
de conservación y la vida de anaquel del producto, facilitando su transporte y almacenamiento (PARK; YADO; BROD, 2001; BRASILEIRO, 1999). Por otro lado, los productos deshidratados utilizan tipos de embalajes más económicos y ofrecen opción de comidas suaves e rápidas (WOODROOF; LUH, 1975). En este sentido el presente trabajo tiene por objetivo obtener datos de la cinética del secado de cupuaçu (Theobroma grandiflorum) a diferentes temperaturas y modelar las curvas del secado a través de los modelos de Fick y de Page.

\section{Materiales y métodos}

El trabajo fue realizado en el Laboratorio de Tecnología de Alimentos de la Universidad Federal de Tocantins - TO, Brasil. La fruta fue adquirida en el comercio local de la ciudad de Palmas, Brasil. Antes del secado se determinó la humedad de las rodajas del producto fresco por el método de secado en estufa hasta valores de peso constante (AOAC, 1990).

\subsection{Cinética del secado}

Para determinar la cinética del secado de las rodajas del cupuaçu (Theobroma grandiflorum), primeramente los frutos fueron lavados en agua corriente y rápidamente fueron secados con papel absorbente y almacenados en congelador a la temperatura de $10{ }^{\circ} \mathrm{C}$ hasta el momento de ser procesados. Para el secado, los frutos fueron retirados del congelador y después de descongelados fueron pelados manualmente y cortados en forma de rodajas con espesor de $0,5 \mathrm{~cm}$.

Las rodajas fueron secadas utilizándose un secador de bandejas de lecho fijo a las temperaturas de 50,60 y $70{ }^{\circ} \mathrm{C}$ y una humedad relativa de aproximadamente $40 \%$. Este secador utilizado es de escala de laboratorio que consta de: una cámara de secado donde son colocadas las bandejas y en cuyas bandejas son colocadas las muestras; un sistema de calentamiento del aire dado por un conjunto de resistencias eléctricas y un sistema de circulación del aire que consta de un ventilador y un anemómetro para el control de la velocidad del aire caliente (GIRALDO-ZUNIGA et al., 2006).

Antes del secado, el secador es colocado en funcionamiento media hora antes para estabilizar la temperatura a la cual será secada la muestra. Una vez estabilizada la temperatura, las muestras fueron colocadas en la bandeja y llevadas para dentro de la cámara del secador, momento que se inicia el proceso.

Durante el secado, las muestras fueron retiradas del secador en intervalos de 1 hora hasta obtenerse el equilibrio dinámico de la muestra con el aire caliente o hasta que no se note cambio en el peso de las muestras (EL-AOUAR; MURR, 2003). El peso de las muestras fue de aproximadamente $45 \mathrm{~g}$, la velocidad del aire de secado fue de $1,5 \mathrm{~m} / \mathrm{s}$ y una humedad relativa del aire variando entre 40 y $50 \%$.

\subsection{Modelo matemático}

Las curvas de secado obtenidas experimentalmente fueron modeladas utilizándose los modelos de Fick (Equação 1) y de Page (Equação 2). El modelo difusional de Fick fue ampliamente utilizada por muchos investigadores en el estudio de secado de alimentos (JAYA; DAS, 2003; EL-AOUAR; MURR, 2003; AGUIRRE; GABITO; CHIRIFE, 1985).

El modelo de Fick (Equação 1) fue utilizado considerándose la muestra como una placa plana infinita, difusividad efectiva constante, sin considerar el encogimiento de la muestra y ausencia de cualquier resistencia al transporte de masa (CRANK, 1975).

$\frac{\mathrm{w}-\mathrm{w}_{\mathrm{e}}}{\mathrm{w}_{0}-\mathrm{w}_{\mathrm{e}}}=\frac{8}{\pi^{2}} \sum_{\mathrm{n}=1}^{\infty} \frac{1}{(2 \mathrm{n}-1)^{2}} \exp \left[-(2 \mathrm{n}-1)^{2} \frac{\pi^{2} \mathrm{D}_{\mathrm{ef}} \mathrm{t}}{4 \mathrm{~L}^{2}}\right]$

$\frac{\mathrm{w}-\mathrm{w}_{\mathrm{e}}}{\mathrm{w}_{0}-\mathrm{w}_{\mathrm{e}}}=$ razón de humedad en base seca, adimensional; $\mathrm{w}$ : humedad media en el instante $\mathrm{t} ;(\mathrm{g} / \mathrm{g}) ; \mathrm{w}_{\mathrm{e}}$ : humedad de equilibrio, $(\mathrm{g} / \mathrm{g}) ; \mathrm{w}_{0}$ : humedad inicial $(\mathrm{g} / \mathrm{g})$; L: espesor medio de la rodaja o placa $(\mathrm{m}) ; \mathrm{D}_{\text {ef }}$ difusividad efectiva, $\left(\mathrm{m}^{2} / \mathrm{s}\right)$; y t: tiempo (s).

El modelo de Page utilizado fue el siguiente (Equação 2):

$\frac{\mathrm{w}-\mathrm{w}_{\mathrm{e}}}{\mathrm{w}_{\mathrm{o}}-\mathrm{w}_{\mathrm{e}}}=\operatorname{Cexp}\left(-\mathrm{Kt}^{\mathrm{n}}\right)$

donde: $\mathrm{w}=$ humedad media en el instante $\mathrm{t}(\mathrm{g} / \mathrm{g}) ; \mathrm{w}_{\mathrm{e}}=$ humedad de equilibrio $(\mathrm{g} / \mathrm{g}) ; \mathrm{w}_{\mathrm{o}}=$ humedad inicial $(\mathrm{g} / \mathrm{g}) ;$ y C, K y $\mathrm{n}=$ parámetros del modelo.

\subsection{Rehidratación}

Una determinada cantidad de rodajas secas con el peso determinado fueron colocadas en cestillos de metal y sumergidas en agua caliente a la temperatura de 50 y $60{ }^{\circ} \mathrm{C}$. A tiempos prefijados las cestillas eran retiradas y escurridas el exceso del agua y se pesaban las muestras hasta obtener valores de pesada constante (MENDOZA, 2003).

\section{Resultados y discusión}

\subsection{Cinética del secado}

El contenido en humedad de las rodajas antes del secado fue de $86,3 \%$. La cinética del secado de un material no es más que la dependencia de la humedad del material y de la intensidad de evaporación con el tiempo o variables relacionadas con este, como la propia humedad o las dimensiones del equipo.

La Figura 1 muestra la variación del contenido de la humedad adimensional del cupuaçu en rodajas en función del tiempo de secado, para las tres temperaturas del aire de secado utilizado. La humedad adimensional fue calculada a partir de la humedad en base seca.

Analizando las curvas de secado en la Figura 1, se observa que la cinética fue fuertemente influenciada por la temperatura y que el empleo de temperaturas mas elevadas redujo significativamente el tiempo necesario para secar la pulpa de cupuaçu, o sea, para un mismo tiempo de proceso, cuanto mayor sea la temperatura del aire, mayor es la velocidad del secado. En la Figura 1 se puede observar también que la pérdida de agua es más rápida al inicio del proceso de secado, siendo que para 
8 horas de secado, el valor del contenido de agua para cada temperatura fue muy diferente, indicando que la temperatura es una variable que influencia bastante el proceso de secado. Este comportamiento fue observado por Prado, Park y Alonso (2000) e Almeida et al. (2002), secando tamaras (Phoenix dactylifera L.) y vainas de algarroba (Ceratonia siliqua), respectivamente. Este acontecimiento ocurre debido a que las temperaturas elevadas del aire producen mayores flujos de secado (HOLDSWORTH, 1986), por causa del correspondiente aumento del coeficiente de convección de transferencia de calor.

También podemos observar que la cinética del secado del cupuaçu tiene características semejantes de la mayoría de los frutos tropicales (MOURA et al., 2001; ARAÚJO et al., 2001). Fue observado que para producir rodajas de cupuaçu con humedad de aproximadamente $20 \%$, fueron necesarias 9,2 horas de secado a $50^{\circ} \mathrm{C}$, siendo que para $60^{\circ} \mathrm{C}$ fue necesario 8,1 horas y 7,3 horas de secado para $70^{\circ} \mathrm{C}$, lo que nos lleva a concluir que será mas ventajoso económicamente deshidratar a $70^{\circ} \mathrm{C}$, pero otros estudios serán necesarios para evaluar la posible pérdida de nutrientes.

\subsection{Modelo matemático}

Las Tablas 1 y 2 muestran los valores de los diferentes parámetros para los modelos de Fick y de Page respectivamente, obtenidos para nuestros datos experimentales de secado del cupuaçu a diferentes temperaturas. El coeficiente de difusividad $\left(D_{\text {ef }}\right)$ es una difusividad efectiva que engloba los efectos de todos los fenómenos que pueden intervenir en la migración o pérdida de agua y su valor es calculado siempre através de modelo matemático ajustando los valores experimentales. Puede entenderse la difusividad como la facilidad con que el agua es removida del material. Como la

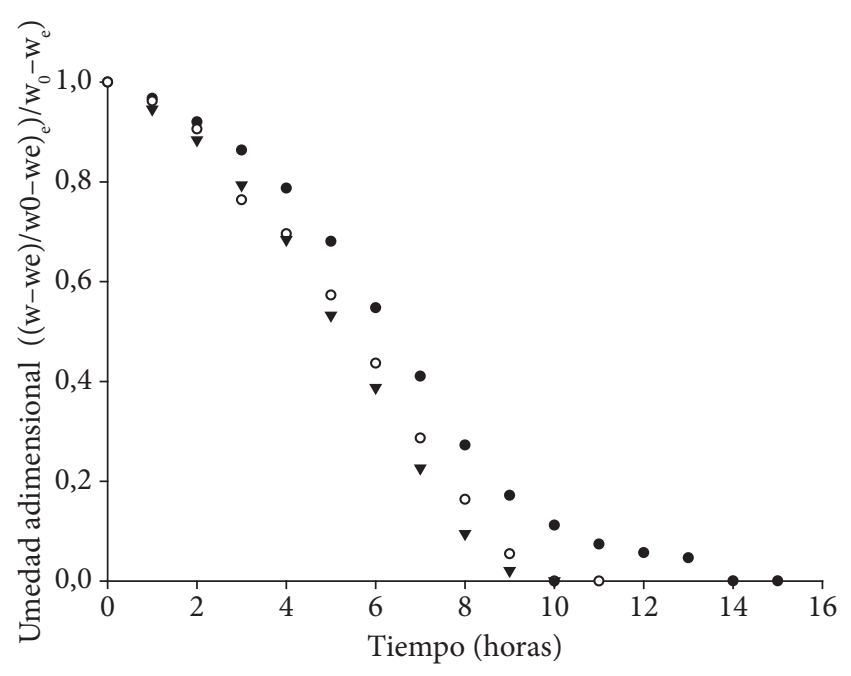

$$
\begin{aligned}
& \text { - Experimental } 50^{\circ} \mathrm{C} \\
& \text { - Experimental } 60^{\circ} \mathrm{C} \\
& \text { - Experimental } 70^{\circ} \mathrm{C}
\end{aligned}
$$

Figura 1. Curvas de secado del cupuaçu para las temperaturas de 50, 60 y $70{ }^{\circ} \mathrm{C}$. difusividad varia conforme cambia las condiciones de secado (temperatura y velocidad del aire), no es intrínseca al material. Por este motivo la llamamos de difusividad efectiva. A través de la Figura 2 y la Tabla 2 se puede verificar que el modelo difusional de Fick, sin considerar el encogimiento de la muestra, fue capaz de predecir la cinética del secado de manera satisfactoria. En la Tabla 2, se puede notar también que para mayores temperaturas del secado mayores son las velocidades del secado, de esta manera, el aumento de la temperatura favoreció la transferencia de masa y como consecuencia el aumento de la difusividad efectiva.

En la Figura 2 puede ser observado que la ecuación de Page se ajusta bien a los datos experimentales, como confirmado por el valor del $\mathrm{R}^{2}$ de la Tabla 2, pudiendo por tanto, ser utilizada en

Tabla 1. Parámetros del modelo de PAGE y coeficiente de determinación $\left(\mathrm{R}^{2}\right)$, calculados por regresión de los datos experimentales de la cinética de secado del cupuaçu.

\begin{tabular}{ccccc}
\hline \multirow{2}{*}{$\begin{array}{c}\text { Temperatura } \\
\text { del aire }\left({ }^{\circ} \mathrm{C}\right)\end{array}$} & \multicolumn{2}{c}{ Parámetros del modelo de PAGE } & \multirow{2}{*}{$\begin{array}{c}\text { Valor } \\
\text { de R }\end{array}$} \\
\hline 50 & $\mathrm{C}$ & $\mathrm{K}$ & $\mathrm{n}$ & \\
\hline 60 & 0,9852 & 0,2918 & 1,1328 & 0,9994 \\
70 & 0,9908 & 0,0279 & 2,0911 & 0,9990 \\
\hline
\end{tabular}

Tabla 2. Coeficientes de Difusión para el modelo Fick.

\begin{tabular}{ccc}
\hline Temperatura del aire $\left({ }^{\circ} \mathrm{C}\right)$ & $\mathrm{D}_{\mathrm{ef}}\left(\mathrm{m}^{2} / \mathrm{s}\right)$ & Valor de $\mathrm{R}^{2}$ \\
\hline 50 & $1,171 \times 10^{-7}$ & 0,9780 \\
60 & $1,273 \times 10^{-7}$ & 0,9630 \\
70 & $1,561 \times 10^{-7}$ & 0,9728 \\
\hline
\end{tabular}

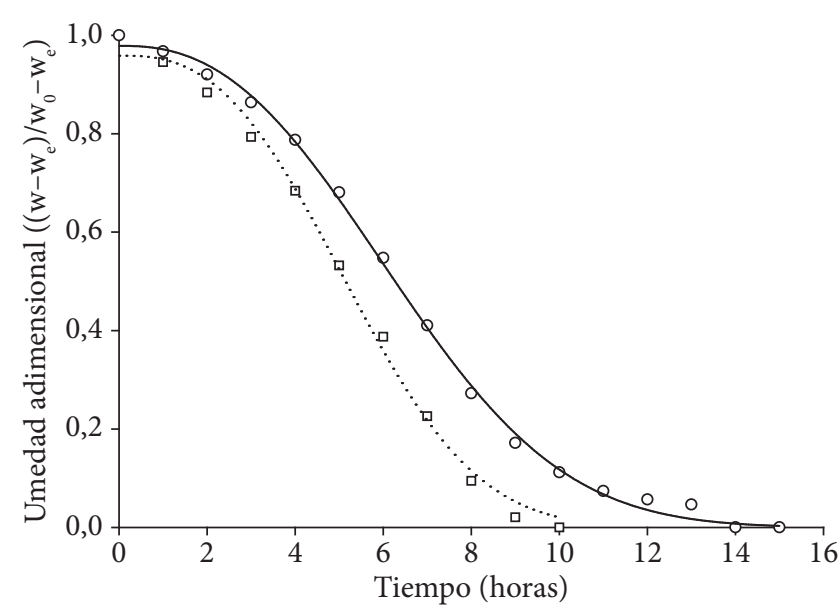
○ Experimental $50^{\circ} \mathrm{C}$
Experimental $70^{\circ} \mathrm{C}$
Modelo $50{ }^{\circ} \mathrm{C}$ Modelo $70{ }^{\circ} \mathrm{C}$

Figura 2. Curvas de secado para las temperaturas de 50 e $70^{\circ} \mathrm{C}$ (datos experimentales y modelados). 


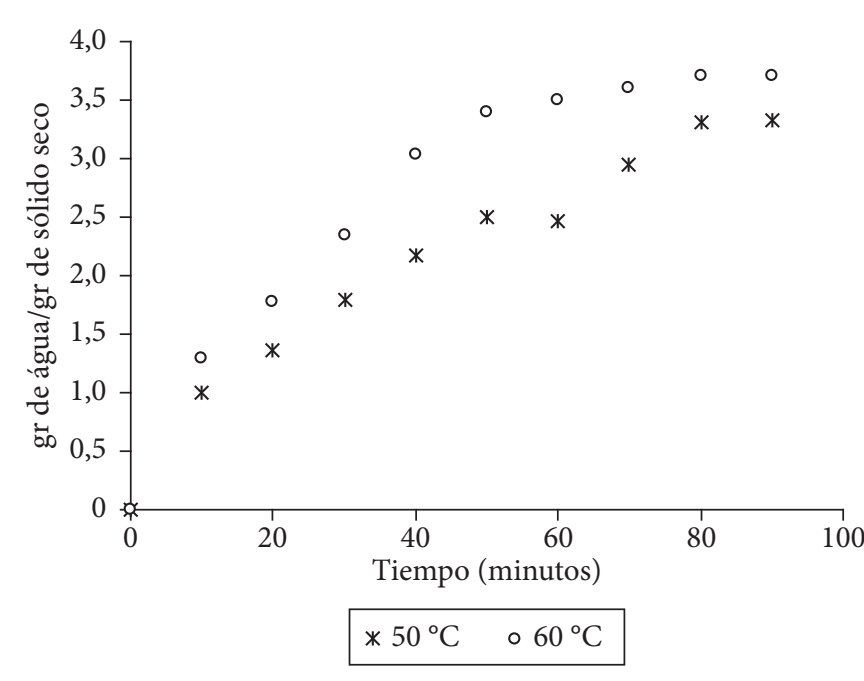

Figura 3. Velocidad de rehidratación del cupuaçu a 50 y $60^{\circ} \mathrm{C}$.

la representación de las curvas de cinética de secado del cupuaçu. Resultados semejantes fueron obtenidos por (GIRALDOZUNIGA, 2004; GABAS; MENEGALLI; TELIS-ROMERO, 1999).

\subsection{Velocidad de rehidratación}

La mayoría de alimentos deshidratados deben ser reconstituidos antes de su consumo, siendo necesario determinar las condiciones óptimas para la rehidratación; en consecuencia, es importante obtener productos rehidratados de textura aceptable en el menor tiempo posible, así, para conhecer a características de rehidratación del cupuaçu fueron realizados los experimentos en dos temperaturas diferentes.

En la Figura 3 se observan las curvas de velocidad de rehidratación correspondientes a las temperaturas 50 y $60^{\circ} \mathrm{C}$, se puede observar que la velocidad de rehidratación fue influenciada directamente por la temperatura de rehidratación.

Resultados semejante fueron obtenidos por Mendoza (2003), para rehidratación de pedazos de papaya.

\section{Conclusiones}

Al comparar las cinéticas de secado del cupuaçu en rodajas, podemos concluir que la cinética del secado fue influenciada fuertemente por la temperatura del aire de secado, temperaturas menores conllevaron a tiempos prolongados de secado para obtener cupuaçu con bajos valores de humedad. Los modelos de Fick y de Page describieron satisfactoriamente los datos experimentales del proceso de secado.

\section{Referencias}

AGUIRRE, R. J.; GABITO, J. F.; CHIRIFE, J. Utilization of Fick's second law for the evaluation of diffusion coefficients in food process controlled by the internal diffusion. Journal of Food Technology, v. 20, n. 22, p. 623-629, 1985.

ALMEIDA, F. A. C. et al. Secagem natural e artificial de vagens de algaroba. Revista Brasileira de Armazenamento, v. 27, n. 1, p. 48-57, 2002.

ARAÚJO, M. E. R. et al. Atividade de água em goiaba (Psidium guajava L.). In: CONGRESSO BRASILEIRO DE ENGENHARIA AGRÍCOLA, 30, 2001, Foz do Iguaçu. Anais... (CD-ROM)

BRASILEIRO, I. M. N. Cinética de secagem de acerola "in natura" em monocamada. Campina Grande, 1999. 80 p. Dissertação (Mestrado) - Universidade Federal da Paraíba - UFPB.

CHITARRA, M. I. F.; CHITARRA, A. B. Pós-colheita de frutos e hortaliças: fisiologia e manuseio. Lavras: ESAL, 1990. 320 p.

CRANK, J. The mathematical of diffusion. 2 ed. Oxford: Clarendon Press, 1975. $414 \mathrm{p}$.

GIRALDO-ZUNIGA, A. D. et al. Kinetic drying experimental data and mathematical model for jackfruit (Artocarpus integrifolia) SLICES. Ciencia y Tecnologia Alimentaria, v. 5, n. 2, p. 89-92, 2006.

GABAS, A. L.; MENEGALLI, F. C.; TELIS-ROMERO, J. Effect of chemical pretreatment on the physical properties of dehydrated grapes. Drying Technology, v. 17, n. 6, p. 1215-1226, 1999.

GOMES, R. P. Fruticultura brasileira. 13 ed. São Paulo: Nobel, 2007. $446 \mathrm{p}$.

HOLDSWORTH, S. D. Advances in the dehydration of fruits and vegetables. In: MACCARTHY, D. (ed.) Concentration and drying of food. London: Elsevier Applied Science Publisher, 1986. p. 293-303.

LORENZI, H. Árvores brasileiras: manual de identificação e cultivo de plantas arbóreas nativas. 2 ed. Nova Odessa: Editora Plantarum, 1998.

MENDOZA, R. Isotermas de sorción y velocidad de rehidratación en frutas de carica papaya $\mathrm{L}$.: deshidratadas con diferentes métodos. Argentina: Universidad Nacional de Misiones, 2003.

MOURA, R. S. et al. Efeito da temperatura e da velocidade do ar na secagem de caju. In: CONGRESSO BRASILEIRO DE ENGENHARIA AGRÍCOLA, 30, 2001, Foz do Iguaçu. Anais... (CD-ROM)

NEVES FILHO, L. G. Alimentos resfriados e congelados. In: CONGRESSO BRASILEIRO DE CIÊNCIA E TECNOLOGIA DE ALIMENTOS, 14, 1994, São Paulo. Anais... São Paulo, 1994. p. 12-19.

PARK, K. J.; YADO, M. K. M.; BROD, F. P. Estudo de secagem de pêra barlett (Pyrus sp.) em fatias. Ciência e Tecnologia de Alimentos, v. 21, n. 3, p. 288-292, 2001.

PRADO, M. E. T.; PARK, K. J.; ALONSO, L. F. T. Shrinkage of dates (Phoenix dactilyfera). Drying Technology, v. 18, n. 1-2, p. 295-310, 2000.

WOODROOF, G. J.; LUH, B. S. Commercial fruit processing. Connecticut: AVI, 1975. p. 375-507. 